\title{
NONLINEAR DIELECTRICS: ELECTROSTATICS OF RANDOM MEDIA AND PROPAGATION OF ELECTROMAGNETIC WAVES IN A HOMOGENEOUS SLAB
}

\author{
Raphael BLUMENFELD and David J. BERGMAN \\ School of Physics and Astronomy, Tel Aviv University, Ramat Aviv, Israel
}

\begin{abstract}
We study nonlinear dielectrics obeying $D=\epsilon\left(E^{2}\right)^{\beta / 2} E$. The solution to the eletrostatic equations is found to be unique for $\infty>\alpha=1 /(\beta+1)>1 /(d-1)$. We find the electrostatic fields produced by a point charge and by an infinite charged slab in such a medium. Two definitions of the effective dielectric constant, $\epsilon_{\text {cff }}$, are shown to coincide. For the case where $\epsilon$ varies in space we show that the differential equation for the first correction to the potential field is dipolar in scaled coordinates. We use the first correction to hound $\epsilon_{\text {eff }}$. Finally we formulate the explicit time dependent equations that describe the dynamic behavior of the magnetic and electric fields in such materials.
\end{abstract}

\section{Electrostatics of the nonlinear dielectric medium}

\subsection{Uniqueness of solution}

Let us assume a nonlinear dielectric medium (NDM) that satisfies [1]

$$
\boldsymbol{D}=\epsilon\left(E^{2}\right)^{\beta / 2} \boldsymbol{E}, \quad \boldsymbol{B}=\mu \boldsymbol{H} \text { and } \boldsymbol{J}=\boldsymbol{\sigma} \boldsymbol{E},
$$

where $\epsilon, \mu$ and $\sigma$ are field independent but may depend on the coordinates. Consider a finite region of space that is occupied by charged conductors at specified potentials and assume there are two different solutions for the field, $\boldsymbol{E}_{i}=-\boldsymbol{\nabla} \Phi_{i}(i=1,2)$ that satisfy the same boundary conditions. The system under study occupies a volume confined between the conductors surfaces and the surface of a sphere of very large radius $R$. Defining

$$
\chi=\left(\boldsymbol{E}_{1}-\boldsymbol{E}_{2}\right) \cdot\left(\boldsymbol{D}_{1}-\boldsymbol{D}_{2}\right)
$$

we integrate over this volume by parts, use Gauss theorem to drop the difference $\operatorname{div}\left(D_{1}-D_{2}\right)$ and note that on the conductors boundaries the surface integral vanishes. Then we are left with 


$$
I_{0}=\int \chi \mathrm{d} v=\oint_{s}\left(\Phi_{2}-\Phi_{1}\right)\left(D_{1}-D_{2}\right) \cdot \mathrm{d} \boldsymbol{S},
$$

where $S$ is the spherical surface at radius $R$. At large $R$ the charged conductors are seen as a point charge which gives rise to a field that satisfies (see below)

$$
|\Phi D| \sim R^{-(2 d-3+(d-2) \beta) /(1+\beta)}
$$

Inserting (1.4) into (1.3) we see that $I_{0}$ vanishes for

$$
\frac{1}{d-1}<\alpha=\frac{1}{\beta+1}<\infty,
$$

where $\alpha$ is the power analogous to the nonlinearity strength in nonlinear resistor networks (NRRNs) [2-8]. Hence the l.h.s. of (1.3) is identically equal to zero. Examining the integrand, $\chi$, we observe that for all functionals $D(E)$ that increase monotonically with $E$, we must have $\chi>0$. This condition is met for all values of $\beta>-1(\alpha>0)$. Therefore for the integral to vanish we must have $E_{1}=E_{2}$ which concludes our proof. Note that this proof is limited to values of $\beta$ that satisfy (1.5). It was found that for nonlinear resistor networks [4] there exist more than one solution for values of $\beta<-1$ (negative values of $\alpha$ ). So in this respect our result is consistent. However, it was shown [3] that a unique solution exists even for $0<\alpha<1 /(d-1)$. Thus we suspect that our proof may be too restrictive and perhaps uniqueness can be shown with a relaxed condition on $\beta$. We would also like to point out that unlike most existing proofs of uniqueness, this proof does not lean on superposition whatsoever.

\subsection{Fields of a point charge and an infinite charged slab in an NDM}

\subsubsection{A point charge}

To find the field induced by a point charge, $q$, in an NDM we use Gauss theorem,

$$
\int D_{\mathrm{p}} \cdot \mathrm{d} S=4 \pi q
$$

and the spherical symmetry, to construct a spherical envelope for the surface of integration in (1.6) to obtain

$$
D_{\mathrm{p}}=q r^{1-d_{\hat{\boldsymbol{r}}}}
$$


where $\hat{r}$ is a unit vector in the radial direction. Consequently we have for the fields

$$
E_{\mathrm{p}}=\left(\frac{q}{\epsilon}\right)^{\alpha} r^{(1-d) \alpha} \hat{\boldsymbol{r}} \quad \text { and } \quad \Phi_{\mathrm{p}}=\frac{(q / \epsilon)^{\alpha}}{(d-1) \alpha-1} r^{1+(1-d) \alpha} .
$$

\subsubsection{An infinite charged insulating slab}

Assume an infinite 3D insulating slab of thickness $t$, lying in the $y-z$ plane that has a charge density $\varphi$ and a dielectric constant $\epsilon_{\mathrm{s}}$ over its volume as opposed to the external one $\epsilon_{\mathrm{e}}$. From symmetry considerations the field is in the $x$-direction, is only $x$-dependent and if we choose the center of the slab as $x=0$ then it is invariant to mirror imaging around the $y-z$ plane. Hence, by using Gauss theorem

$$
D_{\text {in }}(x)=4 \pi \varphi x \quad \text { and } \quad D_{\text {out }}(x)=2 \pi \varphi t \hat{x}
$$

where $\hat{\boldsymbol{x}}$ is a unit vector in the $x$-direction. From (1.9) we find

$$
\boldsymbol{E}_{\mathrm{in}}=\left(\frac{4 \pi \varphi}{\epsilon_{\mathrm{s}}}\right)^{\alpha}|x|^{\alpha \alpha} \hat{\boldsymbol{x}}
$$

and

$$
\begin{aligned}
& \boldsymbol{E}_{\text {out }}=\left(\frac{2 \pi \varphi t}{\epsilon_{\mathrm{c}}}\right)^{\alpha} \hat{\boldsymbol{x}} \\
& \Phi_{\mathrm{in}}=-\left[\frac{4 \pi \varphi}{\epsilon_{\mathrm{s}}}\right]^{\alpha} \frac{|x|^{\alpha+1}}{\alpha+1}
\end{aligned}
$$

and

$$
\Phi_{\text {out }}=(2 \pi \varphi t)^{\alpha}\left[\epsilon_{\mathrm{e}}^{-\alpha}(t / 2-|x|)-\frac{t \epsilon_{\mathrm{s}}^{-\alpha}}{2(\alpha+1)}\right],
$$

where we imposed $\Phi=0$ at $x=0$. The case of a conducting slab can be treated in exactly the same way leading to the same field outside the slab and a zero field inside it.

\section{Inhomogeneous medium}

We define $\varphi$ as the volume charge density and let $\Phi$ vanish on the boundaries of the system. The energy density in the medium is

$$
\delta u=\int \delta \varphi \Phi(\boldsymbol{r}) \mathrm{d} v
$$


Using Gauss theorem and integrating by parts we obtain two integrals, one of which vanishes due to the boundary conditions lcaving us with

$$
\delta u=\frac{1}{4 \pi} \int \boldsymbol{E} \cdot \delta \boldsymbol{D} \mathrm{d} v .
$$

For $\beta>-1$ we can express $\delta \boldsymbol{D}$ in terms of $\boldsymbol{E}$ and after some easy algebraic manipulations one finds

$$
\begin{aligned}
\delta(\boldsymbol{E} \cdot \boldsymbol{D}) & =\boldsymbol{\epsilon}(\beta+2)\left(E^{2}\right)^{\beta / 2}(\boldsymbol{E} \cdot \delta \boldsymbol{E}) \\
& =\frac{\beta+2}{\beta+1} \boldsymbol{E} \cdot \delta \boldsymbol{D} .
\end{aligned}
$$

Inserting (2.3) into (2.2) we identify

$$
u=\frac{1}{4 \pi(\alpha+1)} \int \boldsymbol{E} \cdot \boldsymbol{D} \mathrm{d} v
$$

as the exact energy in our system. In the following we consider an NDM free of charges confined in the $x$-direction between two parallel plates $L$ apart that are held at a constant potential difference, $\Phi_{0}=E_{0} L$ with $\Phi(x=0)=0$. The other boundaries in the perpendicular directions are assumed to be far away and at zero potential. We may define the effective dielectric constant, $\epsilon_{\mathrm{eff}}$, in two different ways:

$$
\langle D\rangle=\epsilon_{\mathrm{eff}}^{(1)}\left(E_{0}^{2}\right)^{\beta / 2} E_{0}
$$

and

$$
\langle U\rangle=\epsilon_{\mathrm{eff}}^{(2)}\left(E_{0}^{2}\right)^{1+\beta / 2} .
$$

We can show that $[1]$

$$
U=\frac{1}{4 \pi(\alpha+1)} \int E_{0} \cdot\langle D\rangle .
$$

Now inserting (2.5) into (2.7) and comparing the result with (2.6) we obtain

$$
\epsilon_{\text {eff }}^{(1)}=\epsilon_{\text {eff }}^{(2)}=\epsilon_{\text {eff }},
$$

i.e., the two definitions are equivalent. The above calculation renders another interesting relation [1]. From (2.6) we have 


$$
4 \pi(\alpha+1)\langle U\rangle=E \cdot\langle D\rangle=\epsilon_{\mathrm{eff}}^{-\alpha}\langle D\rangle^{\alpha+1},
$$

while from (2.4) we obtain

$$
4 \pi(\alpha+1)\langle U\rangle=\langle\boldsymbol{E} \cdot \boldsymbol{D}\rangle=\left\langle\boldsymbol{\epsilon}^{-\alpha} D^{\alpha+1}\right\rangle \text {. }
$$

Comparing the last two expressions we find

$$
\left\langle\epsilon^{-\alpha} D^{\alpha+1}\right\rangle=\epsilon_{\mathrm{eff}}^{-\alpha}\langle D\rangle^{\alpha+1}
$$

As we turn to consider $\epsilon$ that varies with the spatial coordinates we note that there are two exactly solvable geometries.

i) Regions of different dielectric constants whose boundaries lie perpendicular to the capacitor plates. In this configuration $\epsilon$ does not change in the field dircetion and we find

$$
\langle D\rangle=E_{0}^{\beta+1} \sum_{i} p_{i} \epsilon_{i}
$$

where the summation is over the $i$ different components of the system, and where $p_{i}$ is the concentration of the $i$ th component. It follows that

$$
\epsilon_{\mathrm{eff}}=\sum_{i} p_{i} \epsilon_{i}
$$

ii) Slabs of different dielectric constants that lie in parallel to the plates. Now a similar calculation yields

$$
\langle E\rangle=\left(D_{0}\right)^{\alpha} \sum_{i} \epsilon_{i}^{-\alpha}
$$

Hence we find that in this geometry

$$
\epsilon_{\mathrm{eff}}=\left(\sum_{i} p_{i} \epsilon_{i}^{-\alpha}\right)^{-1 / \alpha}
$$

The last two results are analogous to parallel and series summations of conductors in NRRNs.

Now consider diluted media constructed of small homogeneous regions of different values of $\epsilon$, where the distribution of $\epsilon_{i}$ is narrow around $\epsilon_{0}$,

$$
\epsilon(\boldsymbol{r})=\sum_{i} \theta_{i}(r) \epsilon_{i}
$$

and where $\theta_{i}(r)=1$ within the $i$ th region and vanishes outside. We note that $\epsilon_{\mathrm{eff}}$ 
is minimal for a given $\epsilon(r)$ once $E(r)$ is the exact electric field [9]. It then follows that to leading order in $\epsilon(r)-\epsilon_{0}$ the first correction to $\epsilon_{\mathrm{eff}}$ is

$$
\begin{aligned}
\delta \epsilon_{\mathrm{eff}} & =\frac{1}{V} \int\left(\epsilon(r)-\epsilon_{0}\right)\left[\frac{|E(r)|}{E_{0}}\right]^{\beta+2} \mathrm{~d} v \\
& =\sum_{i} p_{i} \epsilon_{i}-\epsilon_{0} .
\end{aligned}
$$

Relying again on this minimum property [9] then some manipulations [1] lead to the second correction,

$$
\delta^{2} \epsilon_{\mathrm{eff}}=\frac{(\beta+2) E_{0}^{-(\beta+2)}}{(d-2) \Omega_{d}(\alpha+1) V} \sum_{i} \int \theta_{i}(r) \delta \epsilon_{i}|E(r)|^{\beta}|E \cdot \delta E| \mathrm{d} v .
$$

Hence we need only the first order correction to the field $\delta E$. From div $D=0$ and assuming $E \not \equiv 0$, we get

$$
E^{2}(\epsilon \operatorname{div} E+E \cdot \nabla \epsilon)+\beta \epsilon \sum_{j, k} E_{j} E_{k} \frac{\partial E_{k}}{\partial x_{j}}=0 .
$$

Expanding $E$ and $\epsilon$ to first order, using Gauss theorem again and substituting for the unperturbed field yields a differential equation for $\delta E$. Transforming this equation to rescaled coordinates $y_{t}^{\prime}=y_{t}, x^{\prime}=x / \sqrt{1+\beta}$ and substituting into (2.15) we obtain

$$
-\nabla^{\prime 2} \delta_{1} \phi=-\frac{\sqrt{\alpha} E_{0}}{\epsilon_{0}} \frac{\partial}{\partial x^{\prime}} \delta_{1} \epsilon
$$

where div' is the divergence taken in the primed coordinates. Eq. (2.16), however, is exactly Poisson's equation for the potential field produced by a sheet of electric dipoles at the internal interfaces.

Now we can solve this linear problem as in the literature [9] to find the first order contribution to $\delta^{2} \epsilon_{\text {eff }}$,

$$
\begin{aligned}
\delta^{2} \eta_{\mathrm{eff}}= & \frac{1}{(d-2) \Omega_{d} V^{\prime}} \sum_{\mu, \nu \in V_{\mu}} \int \theta_{\mu}\left(r^{\prime}\right) \theta_{\nu}\left(\rho^{\prime}\right) \delta \eta_{\mu} \delta \eta_{\nu} \\
& \times \frac{\partial^{2}}{\partial x^{\prime 2}}\left[\frac{1}{\left|\boldsymbol{r}^{\prime}-\rho^{\prime}\right|^{d-2}}\right] \mathrm{d}^{d} r^{\prime} \mathrm{d}^{d} \rho^{\prime},
\end{aligned}
$$

where $\eta=\epsilon / \epsilon_{0}, \Omega_{d}$ is the surface area of a unit sphere in $d$-dimensions, the sum runs over all the grains and $V_{\mu}$ is a spherical volume around the $\mu$ th grain that contributes to the near field in it. Partitioning the sums within given 
regions of different dielectric constants, so that $\mu \in i$ and $\nu \in j$, one finds

$$
\delta^{2} \eta_{\text {eff }}=\frac{1}{(d-2) \Omega_{d} V^{\prime}} \sum_{i, j} \int g_{i j} \frac{\partial^{2}}{\partial x^{\prime 2}}\left[\frac{1}{\left|\boldsymbol{r}^{\prime}-\rho^{\prime}\right|^{d-2}}\right] \mathrm{d}^{d} r^{\prime} \mathrm{d}^{d} \rho^{\prime},
$$

where $g_{i j}$ is the partially summed correlation function

$$
g_{i j}\left(\boldsymbol{r}^{\prime}, \boldsymbol{\rho}^{\prime}\right)=\sum_{\mu \in i} \sum_{\nu \in j \text { in } v_{\mu}} \theta_{\mu}\left(\boldsymbol{r}^{\prime}\right) \theta_{\eta}\left(\boldsymbol{\rho}^{\prime}\right) .
$$

The difference between this case and the linear one now follows from the fact that while there $g_{i j}$ was spherically symmetric, here it has an elliptic symmetry. So we can bound the near field contribution to the second correction and consequently, after evaluating the far field contribution we find an exact lower bound for $\delta^{2} \eta_{\text {eff }}$ and hence for $\epsilon_{\text {eff }}=\epsilon_{0}\left(1+\delta \eta+\frac{1}{2} \delta^{2} \eta\right)$

$$
\begin{array}{ll}
\sum_{i} p_{i} \delta \eta_{i}\left[\sum_{j} p_{j} \delta \eta_{j}-\delta \eta_{i}\right] \leqslant \frac{d}{2} \delta^{2} \eta_{\text {eff }}, & \beta \leqslant 0, \\
\sum_{i} p_{i} \delta \eta_{i}\left[\sum_{j} p_{j} \delta \eta_{j}-\delta \eta_{i}\right] \geqslant \frac{d}{2} \delta^{2} \eta_{\text {eff }}, & \beta \geqslant 0 .
\end{array}
$$

\section{Propagation of wave in an NDM}

To study the electrodynamic behavior within an NDM we chose the geometry of an infinite slab of thickness $W$ (in the $z$-direction) embedded in vacuum and a source outside it. We set the axes such that

$$
\boldsymbol{E}=E \hat{\boldsymbol{x}} \quad \text { and } \quad \boldsymbol{H}=H \hat{\boldsymbol{y}} \text {, }
$$

where $E$ and $H$ are scalar functions of $z$ and the time only. By Maxwell's equations and via textbooks derivation we have

$$
\begin{aligned}
& -\frac{\partial H}{\partial z}=\frac{\partial D}{\partial t}+\sigma E, \\
& -\frac{\partial E}{\partial z}=\mu \frac{\partial H}{\partial t},
\end{aligned}
$$

where $\mu$ is assumed constant in the entire space. It follows that

$$
\frac{\partial^{2} E}{\partial z^{2}}=\mu \frac{\partial^{2} D}{\partial t^{2}}+\sigma \mu \frac{\partial E}{\partial t} .
$$


Eqs. (3.2) and (3.3) in addition to appropriate boundary conditions determine the transmitted and reflected waves. By applying standard boundary conditions considerations one has for the fields outside the slab

$$
H(z, t)=\sqrt{\frac{\epsilon_{0}}{\mu}} E(z, t) .
$$

Defining the function

$$
M(E)=\sqrt{\epsilon \mu(1+\beta)}|E|^{\beta / 2},
$$

we look for a solution for $E$ that has the form

$$
E=G\left[t-\left(z-z_{0}\right) M(E)+t_{0}\right],
$$

where $t_{0}$ is a constant and $z_{0}$ is some point that lies outside the slab in the region $z<0$. Assuming $G(U)$ is differentiable at least once, we find

$$
\frac{\partial E}{\partial z}=-M(E) \frac{\partial E}{\partial t} .
$$

Assuming $\sigma=0$ we can now find $H$ as a function of $E$ :

$$
H=\left[\frac{\epsilon(\beta+1)}{\mu}\right]^{1 / 2} \frac{E^{\beta / 2}}{1+\beta / 2} E,
$$

which can be verified by differentiation and using (3.7) to compare with the direct derivatives (3.2) and (3.3). Rewriting (3.4)

$$
\frac{\partial^{2} E}{\partial z^{2}}=\mu \epsilon(\beta+1)\left[E_{i} \frac{\partial^{2} E}{\partial t^{2}}+\beta\left(\frac{\partial E_{i}}{\partial t}\right) \frac{\partial E}{\partial t}\right] E_{i}|E|^{\beta-2},
$$

we find the two equations that govern the electromagnetic behaviour of a wave in an NDM.

To conclude, we studied continuous media that obey a power-law relation between $\boldsymbol{D}$ and $\boldsymbol{E}$. We have found the static potential field generated by a point charge and by an infinite slab within such homogeneous media. We have shown that the static solution is unique for $1 /(d-1)<\alpha<\infty$ where $\alpha$ is the nonlinearity strength, which agrees almost completely with known results found for the conductivity of nonlinear resistor networks [3,4]. For $0<\alpha<1 /(d-1)$ we were not able to prove uniqueness, although the solution is such for finite size systems [11] in this range. Next we introduced two definitions for $\epsilon_{\text {eff }}$ in inhomogeneous media via $\langle D\rangle$ and $\langle U\rangle$ and showed them to be equivalent. 
We found the corrections to the fields and bounded the effective dielectric constant for all $\alpha$. We think our method is the only one available for treating $\epsilon_{\text {eff }}$ in a strongly nonlinear material because it does not use the superposition principle, unlike existing methods [10] that lean heavily on superposition, and are very difficult to extend in a straightforward way to nonlinear media. Then we turned to dynamic fields. We introduced the generalised equations that one has to solve and found the explicit solution for $H(E)$.

\section{References}

[1] Most of the following results are derived in a more extensive work by R. Blumenfeld and D.J. Bergman, submitted for publication.

[2] S.W. Kenkel and J.P. Straley, Phys. Rev. Lett. 49 (1982) 767;

J.P. Straley and S.W. Kenkel, Phys. Rev. B 29 (1984) 6299.

[3] R. Blumenfeld and A. Aharony, J. Phys. A: Math. Gen. 18 (1985) L443.

[4] R. Blumenfeld, Y. Meir, A.B. Harris and A. Aharony, J. Phys. A: Math. Gen. 19 (1986) L791.

[5] Y. Meir, R. Blumenfeld, A. Aharony and A.B. Harris, Phys. Rev. B 34 (1986) 3424.

[6] Y. Meir, R. Blumenfeld, A. Aharony and A.B. Harris, Phys. Rev. B 36 (1987) 3950.

[7] R. Rammal and A.-M.S. Tremblay, Phys. Rev. Lett. 58 (1987) 415.

[8] R. Blumenfeld, J. Phys. A 21 (1988) 815.

[9] D.J. Bergman, Phys. Rep. 43 (1978) 377; D.J. Bergman, bulk physical properties of composite media in: Les Methodes de l'Homogeneisation Theorie et Applications en Physique (Editions Eyrolles, Paris 1985).

[10] D. A. Bruggeman, Ann. Phys. Leipzig 29 (1937) 160; S. Kirkpatrick, Phys. Rev. B 15 (1977) 1533; R. Landauer, in AIP Conf. Proc. No. 40, C.W. Garland and D. Tanner, eds. (AIP, New York, 1978) and references therein.

[11] R. Blumenfeld, Ph.D. Thesis. 